\title{
Heterologous Overexpression of Sup35 in E. coli Leads to Both Monomer and Complex States
}

\author{
Mingyang Wang ${ }^{1}$, Xiao Wang ${ }^{1}$, and Zhenyun Cheng ${ }^{1}$ \\ ${ }^{1}$ Clinical Laboratory The First Affiliated Hospital of Zhengzhou University Key Clinical \\ Laboratory of Henan Province Zhengzhou Henan 450052 China
}

December 31, 2021

\begin{abstract}
The heterologous overexpression states of prion proteins play a critical role in understanding the mechanisms of prion-related diseases. We report herein the identification of soluble monomer and complex states for a bakers' yeast prion, Sup35, when expressed in E. coli. Two peaks are apparent with the elution of His-tagged Sup35 by imidazole from a Ni ${ }^{2+}$ affinity column. Peak I contains Sup35 in both monomer and aggregated states. Sup35 aggregate is abbreviated as C-aggregate and includes a non-fibril complex comprising Sup35 aggregate-HSP90-Dna K, ATP synthase $\beta$ unit (chain D), 30S ribosome subunit, and Omp F. The purified monomer and C-aggregate can remain stable for an extended period of time. Peak II contains Sup35 also in both monomer and aggregated (abbreviated as S-aggregate) states, but the aggregated states are caused by the formation of inter-Sup35 disulfide bonds. This study demonstrates that further assembly of Sup35 non-fibril C-aggregate can be interrupted by the chaperone repertoire system in E. coli.
\end{abstract}

Heterologous Overexpression of Sup35 in E. coli Leads to Both Monomer and Complex States Mingyang Wang ${ }^{\mathrm{a}}$, Xiao Wang ${ }^{\mathrm{a}}$, and Zhenyun Cheng ${ }^{\mathrm{a} *}$

${ }^{a}$ Clinical Laboratory, The First Affiliated Hospital of Zhengzhou University, Key Clinical Laboratory of Henan Province, Zhengzhou, Henan 450052, China. Fax: 0371-66278235.

*E-mail address: czc828026@163.com

\begin{abstract}
The heterologous overexpression states of prion proteins play a critical role in understanding the mechanisms of prion-related diseases. We report herein the identification of soluble monomer and complex states for a bakers' yeast prion, Sup35, when expressed in E. coli . Two peaks are apparent with the elution of His-tagged Sup35 by imidazole from a $\mathrm{Ni}^{2+}$ affinity column. Peak I contains Sup35 in both monomer and aggregated states. Sup35 aggregate is abbreviated as C-aggregate and includes a non-fibril complex comprising Sup35 aggregate-HSP90-Dna K, ATP synthase $\beta$ unit (chain D), 30S ribosome subunit, and Omp F. The purified monomer and C-aggregate can remain stable for an extended period of time. Peak II contains Sup35 also in both monomer and aggregated (abbreviated as S-aggregate) states, but the aggregated states are caused by the formation of inter-Sup35 disulfide bonds. This study demonstrates that further assembly of Sup 35 non-fibril C-aggregate can be interrupted by the chaperone repertoire system in E. coli .
\end{abstract}

Keywords:

Sup35; heterologous overexpression states; chaperone proteins; non-fibril aggregates

Introduction 
The prion-folding states orchestrated by chaperone systems in protein homeostasis are closely related to prion diseases, such as transmissible spongiform encephalopathy (TSE) [1-3]. Previous studies have revealed that prion transmission is a universal phenomenon, which shows intriguingly similar self-assembly behavior and heredity stability from $E$. coli and yeast to mammals [4-6].However, cells in different organisms exhibit distinct responses and regulatory mechanisms for prion assembly [7]. Investigations on the heterologous expression of a prion protein in a foreign host should allow one to gain insight into the prion self-assembly mechanism.

S. cerevisiae Sup35 protein is an ideal model for examining prion self-assembly and genetic stability from parental cells to filial cells. In its normal monomer state, Sup35 serves as a translation termination release factor, dictated by the C-terminal $[8,9]$. In contrast, the N-terminal, comprising relatively conserved amino acids, serves as the core functional domain for amyloid formation [10]. Sup35-NM, which was constructed using the N-terminal and middle (M) domain, is typically chosen for in vitro self-assembly studies. Transforming in vitro -prepared amyloid fibrils into non-prion state yeast cells can effectively induce prion state phenotypes, and these phenotypes possess genetic stability from one generation to the next [11,12]. Meanwhile, Sup35 overexpression in yeast causes the prion state phenotype $\left[P S I^{+}\right][13]$, which limits the investigation of prion transmission in the in vivo state by homologous overexpression. HSP104 has been suggested as a contributing factor in the development of prion states in $\left[P S I^{+}\right][14,15]$, but the process by which exhaustive genetic and self-assembly stability is maintained in yeast cells has remained unclear.

Many conclusions about the in vitro prion self-assembly mechanism have been drawn from prokaryotic purified proteins [16-18]. Examinations of Sup35 heterologous expression have primarily focused on two aspects. For one, GPI-anchored Sup35 in mammalian cells can form aggregates, which are different from the fibrils assembled in vitro $[19,20]$. This suggests that heterologous expression is feasible and can contribute to the understanding of the prion self-assembly mechanism. In addition, studies examining Sup35 and Sup35NM self-assembly have been conducted in E. coli . Sup35-NM fusion expression with green fluorescent protein in E. colishowed bright foci and amyloid-like structures [21]. Several lines of evidence have shown that overexpressed Sup35 could be purified in soluble states in E. coli [22,23]. In contrast, Sup35 from yeast formed high molecular weight aggregates. These findings suggest that Sup35 may exist in the cytoplasm of E. coli cells as monomers. The cellular components responsible for maintaining the monomer state for Sup35 in heterologous E. coli are not fully understood. Furthermore, whether Sup35 monomer constitutes the sole surviving state in E. coli remains unclear. Answering these questions will provide a solid foundation for achieving prion self-assembly in a controlled fashion and can offer deeper insight into prion in vivo heredity and infectivity.

\section{Materials and methods}

\section{Strains and culture}

Sup35 was cloned from 74-D694 [ $\left.\mathrm{psi}^{-}\right]$genomic DNA. Restriction endonuclease digestion sites, Not I and Nco I, were chosen as Sup35 insertion sites for the pET28a expression vector. The primer sequences were as follows: Sup35-Forward, 5'-GTGCCGCGCGGCAGCCATATGTCGGATTCAAACCAAGGC-3'; Sup35- Reverse, 5'-TGGTGGTGCTCGAGTGCGGCCGCTTACTCGGCAATTTTAACAAT -3'. The target pET28aSup35 vector was amplified in DH5 $\alpha$ (transformed by traditional heat-shock transfer method) and sequenced for the verification of an as-designed sequence. BL21 Rosseta (DE3) cells harboring the pET28a-Sup35 vector were used for the expression and purification of Sup35. The transformants were cultured in LB medium with $50 \mathrm{mg} / \mathrm{L}$ kanamycin at $37^{\circ} \mathrm{C}$ and $220 \mathrm{rpm}$. When these cells' $\mathrm{OD}_{600}$ reached 1.0, IPTG was added to the medium with a final concentration of $0.5 \mathrm{mM}$. The growing condition for these transformants was then switched to $30^{\circ} \mathrm{C}$ and $160 \mathrm{rpm}$. After $4 \mathrm{~h}$, the cells were harvested at $4^{\circ} \mathrm{C}$ and $5000 \mathrm{~g}$ for $5 \mathrm{~min}$.

The yeast was of the 74-D694 [ $\mathrm{psi}^{-}{ }^{-}$strain. The cells were cultured with YPAD medium $(20 \mathrm{mg} / \mathrm{mL}$ Ade) for $48 \mathrm{~h}$.

\section{Purification of Sup35 protein}


Sup35 induced cells were resuspended in lysis buffer (buffer A, $20 \mathrm{mM}$ Tris, $\mathrm{pH}$ 8.0, $1.2 \mathrm{M} \mathrm{NaCl}, 10 \mathrm{mM}$ PMSF). The bacterial suspension was then sonicated to lyse the cells. The $50 \mathrm{~mL}$ lysis mixture from the 1.5 $\mathrm{L}$ total culture was centrifuged at $8000 \mathrm{~g}$ for $15 \mathrm{~min}$, and the supernatant was filtered by $0.22 \mu \mathrm{m}$ membranes. All the purification processes were conducted with GE AKTA purifier (pH/c-900, UV-900, P-900, frac-900) at $4^{\circ} \mathrm{C}$. An $\mathrm{Ni}^{2+}$-NTA agarose column (His Trap HP, 175248-01) was used for first-stage purification. The column was washed with wash buffer $(20 \mathrm{mM}$ Tris, $\mathrm{pH} 8.0,20 \mathrm{mM}$ imidazole, $10 \mathrm{mM}$ PMSF, $5 \%$ buffer B) for 20 column volumes (flow rate: $1.5 \mathrm{~mL} / \mathrm{min}$ ). The target protein was eluted by $5-50 \%$ and $50-100 \%$ imidazole gradients with buffer B (20 mM Tris, pH 8.0, $10 \mathrm{mM}$ PMSF, $500 \mathrm{mM}$ imidazole, $1.2 \mathrm{M} \mathrm{NaCl})$. Protein concentration was measured using the BCA protein assay kit (Pierce, product \#23227).

\section{Size exclusion chromatography (SEC) of Sup35 complexes and monomers}

Freshly prepared Sup35 using an $\mathrm{Ni}^{2+}$-NTA agarose column was dialyzed with buffer C (20 mM Tris, pH 8.0, $1.2 \mathrm{M} \mathrm{NaCl}$ ) for $12 \mathrm{~h}$ to remove imidazole. Subsequently, the sample was injected into a HiLoad Superdex $200 \mathrm{pg}$ 16/60 size exclusion chromatographic (SEC) column (1 mL/min, 17-1069-01) for peak I separation (flowing phase, buffer C). For the second SEC separation, the eluted samples were stored for 14-day at $4^{\circ} \mathrm{C}$ without concentration. For SDS-PAGE and semi-denaturating detergent agarose gel electrophoresis, the samples were concentrated by $2500 \mathrm{~g}$ centrifugation with an ultrafiltration tube (Millipore, $3000 \mathrm{MWCO}$ ).

\section{TEM}

An imaging-ready carbon-coated copper grid was placed on the sample and remained there for 3 min. The grid was then stained with a $2 \%$ uranyl formate aqueous solution (containing $25 \mathrm{mM} \mathrm{NaOH}$ ) for 3 min and was subsequently washed two times with distilled water. TEM imaging was performed at $100 \mathrm{kV}$.

Yeast Sup35 monomer release and se mi-denaturating detergent agarose gel electrophoresis (SDD-AGE) [24]

$1.2 \%$ agarose solution $(40 \mathrm{~mL})$ in $1 \times$ TAE was melted in a microwave oven, and $400 \mu \mathrm{L} 10 \%$ SDS was added to the gel solution. Subsequently, this mixture was carefully cast into the slab to avoid any bubbles in the gel. After the gel had set, the slab was placed in the electrophoresis chamber (Bio-Rad, Mini sub-cell) and submerged in $1 \times$ TAE containing $0.1 \%$ SDS at $20 \mathrm{~V}$ for $4 \mathrm{~h}$.

The release of Sup35 monomers from the 74-D694 [psi-] cells was performed using the lyticase method. Cell lysates or purified Sup35 protein ( $40 \mu \mathrm{L}$ each) was immediately mixed with $6 \times$ loading buffer (300 $\mathrm{mM}$ Tris $\mathrm{pH} 6.8,60 \%$ glycerol, $0.6 \%$ bromophenol blue) and loaded into an agarose gel for SDD-AGE. Subsequently, the gels were used for western blot.

\section{Western blot assay}

TBS-T buffer (0.05\% Tween-20) was used for the western blot assay. SDD-AGE and SDS-PAGE gels were transferred onto a nitrocellulose membrane (PALL) with transfer buffer (25 mM Tris, pH 8.0, $200 \mathrm{mM}$ glycine, $20 \%$ methanol). Then, the membrane was washed 3 times with TBS buffer and was blocked by $5 \%$ non-fat powdered milk in TBS-T ( $5 \%$ non-fat powered milk/TBS-T, $1 \mathrm{~h}$ at room temperature). The membrane was subsequently washed 3 times with TBS-T buffer and was probed with anti-Sup35 sequence-specific antibody (Santa Cruz, sc25915) (1:5000 dissolved in 5\% non-fat powered milk/TBS-T) for 30 min at room temperature, which was followed by 3 washes with TBS-T, each for 5 min. A secondary antibody (1:8000) conjugated with HRP was then incubated on the membrane for $30 \mathrm{~min}$ at room temperature. After being washed 3 times with TBS-T, the membrane was incubated with ECL solution (Pierce, product \#34095), and 1 min later, $\mathrm{X}$-ray film imaging was conducted in the dark room.

Blue native polyacrylamide gel electrophoresis(BN-PAGE) [25], SDS-PAGE and protein identification by MALDI-TOF/TOF

The ingredients for the preparation of the BN-PAGE gel were the same as those used for SDS-PAGE, except that preparation of the BN-PAGE gel was conducted in the absence of SDS. The basic (anode) buffer contained $20 \mathrm{mM}$ Tris and $250 \mathrm{mM}$ glycine. In addition to the ingredients in the basic buffer, the 
cathode buffer contained an additional ingredient, $0.1 \%$ Coomassie brilliant blue G250. At the beginning of the electrophoresis, an $80 \mathrm{~V}$ was applied for $20 \mathrm{~min}$. A $20 \mathrm{~mA}$ constant current was used throughout the rest of the process (for approximately $1.5 \mathrm{~h}$ ). The gel was then directly immersed in the destaining solution (distilled water:ethanol:acetic acid $=5: 4: 1$ ) for $30 \mathrm{~min}$.

The samples for SDS-PAGE were boiled with $6 \times$ SDS loading buffer (300 mM Tris pH 6.8, $60 \%$ glycerol, $12 \%$ SDS, $0.6 \%$ bromophenol blue). To eliminate disulfide bonds, reducing agent (10 mM DTT) was added prior to boiling. All the gel concentrations used for SDS-PAGE and BN-PAGE were $12 \%$.

The dissociated bands of compound I were excised from SDS-PAGE, which were digested with trypsin and then identified by matrix-assisted laser desorption ionization-time of flight (MALDI-TOF) mass spectrometry. The MS plus MS/MS search was performed using the MASCOT search engine v3.5 (Matrix Science Ltd., London).

\section{Results and Discussion}

Sup35 was consistently eluted in a stepwise manner. Two peaks were observed from non-denatured $\mathrm{Ni}^{2+}$ affinity chromatography (Fig 1A). Peak I was dissociated into three main bands on SDS-PAGE (Fig 1B), the Sup35 monomer band, an undetermined band (approximately $45 \mathrm{kDa}$, named as $\mathrm{X} 1$ ), and an outer membrane protein band (Omp F) [26]. The assignment of Omp F is based on its molecular weight shown on SDS-PAGE, the intensity of the band and the mass spectrum result (Table S1). The dissociation of peak I on SDS-PAGE had many continuous bands between the monomer and X1, which were defined as zone X2 (Fig 1B). Peak II exhibited a monomer band on SDS-PAGE with an intensity less than that under DTT. This indicates that part of the species in peak II is aggregated (S-aggregate) and caused by inter-Sup35 disulfide bonds. No intensity change could be observed for the protein bands associated with peak I on SDS-PAGE with the addition of DTT, thus excluding the formation of inter-Sup35 disulfide bonds.

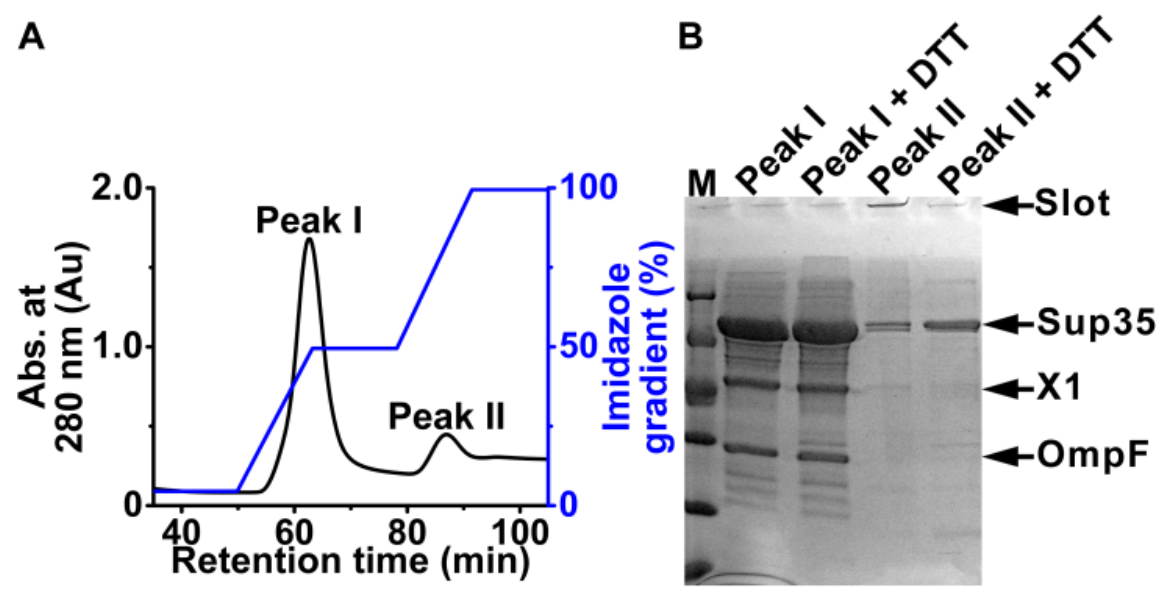

Fig 1. Purification and SDS-PAGE of overexpressed Sup35 inE. coli under non-denatured $\mathrm{Ni}^{2+}$ affinity chromatography. (A) Elution curve of Sup35 under non-denatured $\mathrm{Ni}^{2+}$ affinity chromatography. Peak I and peak II appeared in approximately 5-50\% and 50-100\% imidazole gradients, respectively. The buffers used here were buffer A (20 mM Tris, pH 8.0, 1.2 M NaCl, $10 \mathrm{mM}$ PMSF, 0\% imidazole) and buffer B (20 mM Tris, pH 8.0, 1.2 M NaCl, $10 \mathrm{mM}$ PMSF, $500 \mathrm{mM}$ imidazole, 100\%). (B) SDS-PAGE for the fractions in Figure 1A. All lanes were treated by SDS boiling. Slot represents for sample loading slots in stacking gel. Sup35, X1 and OmpF indicate the bands of Sup35 monomer, X1 protein and OmpF, respectively. Peak I + DTT and Peak II + DTT were boiled with $10 \mathrm{mM}$ DTT. Lane M: molecular weight protein marker comprising $116 \mathrm{kDa}, 66.2 \mathrm{kDa}, 45 \mathrm{kDa}, 35 \mathrm{kDa}, 25 \mathrm{kDa}$, and $18.4 \mathrm{kDa}$. 


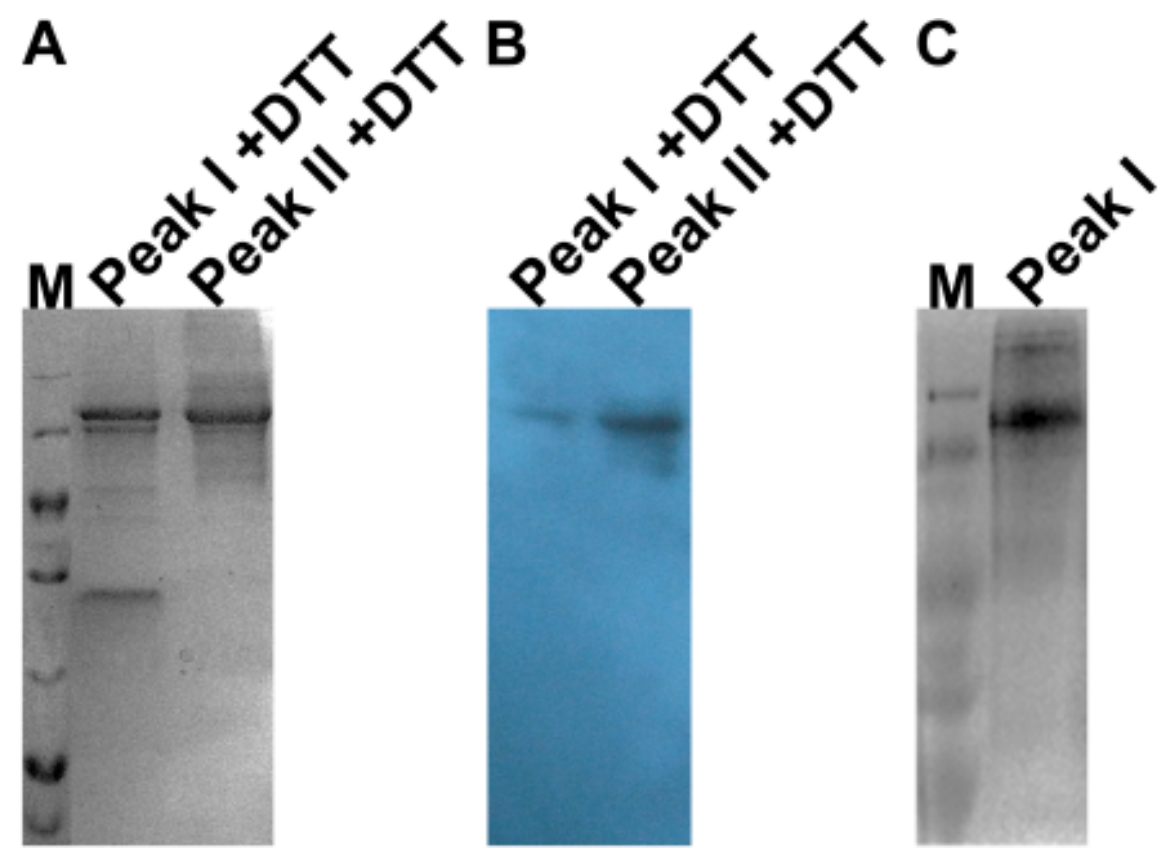

Fig 2. Western blot and BN-PAGE analyses of peak I and II. (A) SDS-PAGE of peak I (lane 1) and peak II (lane 2) under DTT. (B) Western blot of peak I and peak II, with DTT, using Sup35 sequencespecific antibody. (C) BN-PAGE analysis of peak I. Peak I + DTT and Peak II + DTT all had the same amount of total protein in Figures $2 \mathrm{~A}$ and $2 \mathrm{~B}$. The amount of protein in Figure $2 \mathrm{C}$ was twice as much as that in Figure 2A. Lanes $\mathrm{M}$ in Figures $2 \mathrm{~A}$ and 2C: molecular weight protein marker comprising $116 \mathrm{kDa}$, $66.2 \mathrm{kDa}, 45 \mathrm{kDa}, 35 \mathrm{kDa}, 25 \mathrm{kDa}, 18.4 \mathrm{kDa}$, and $14.4 \mathrm{kDa}$.

Due to the relatively high molecular weight of Sup35 (685 amino acids, 5 cysteine residues, $76.6 \mathrm{kDa}$ as calculated from the amino acid sequence), Sup35 fused with histidine (His-tag) in the C-terminal was chosen for the elimination of partially translated Sup35 (Fig S1). Western blot with sequence-specific antibody shows that the band between $116 \mathrm{kDa}$ and $66.2 \mathrm{kDa}$ was the Sup35 monomer band, while the other dissociated bands had no signal (Figs 2A and 2B). This indicates that peak I comprises a monomer and other proteins, with the whole complex interacting with $\mathrm{Ni}^{2+}$ affinity chromatography through the His-tag. Meanwhile, peak I exhibited a monomer band and high molecular weight bands (including a band at the loading slot) in blue native polyacrylamide gel electrophoresis (BN-PAGE) and could not be disaggregated to form clear $\mathrm{X} 1$ and Omp F bands (Fig 2C). This was the foundation of the Sup35 monomer SEC purification (Fig 3). All these conclusions were prerequisite for the Sup35 monomer and complex research below. 
A

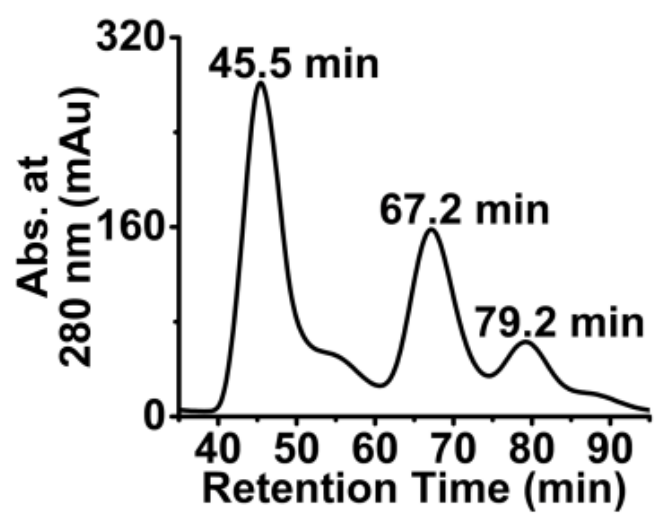

B

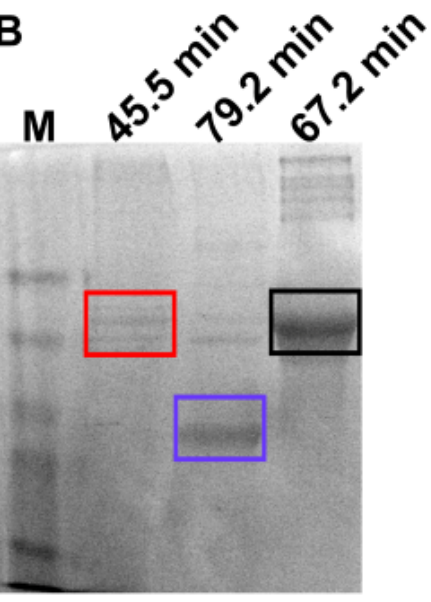

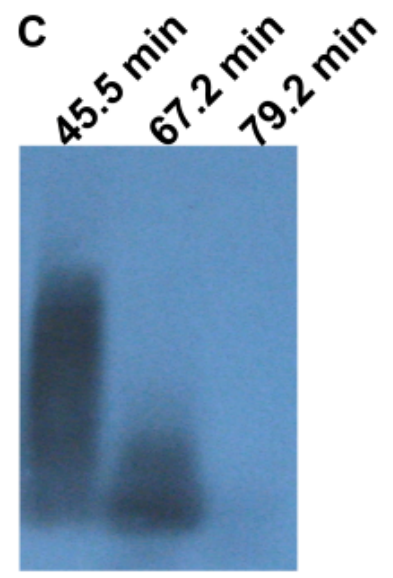

D

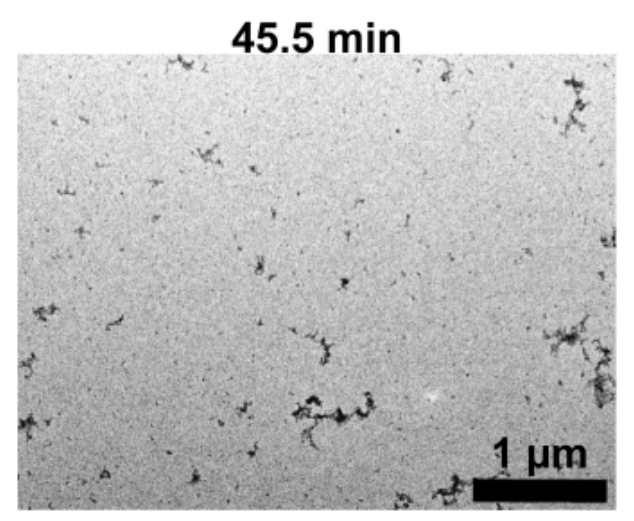

Fig 3. Dissection of peak I and subsequent analysis.(A) Size exclusion chromatography (SEC) curve of peak I. Imidazole was removed from the peak I sample using dialysis. $45.5 \mathrm{~min}, 67.2 \mathrm{~min}$, and $79.2 \mathrm{~min}$ represented the different retention times of the fractionated species. Buffer $\mathrm{C}$ was used here $(20 \mathrm{mM}$ Tris, $\mathrm{pH}$ 8.0, 1.2 M NaCl). (B) SDS-PAGE for $45.5 \mathrm{~min}, 67.2 \mathrm{~min}$, and $79.2 \mathrm{~min}$ fractions in Figure 3A. The bands in black, blue, and red frames were selected for mass spectrometry. (C) SDD-AGE of peak I SEC fractions. (D) Transmission electron microscopy (TEM) of 45.5 min species from Figure 3A. Lane M in Figure 3B: molecular weight protein marker comprising $100 \mathrm{kDa}, 70 \mathrm{kDa}, 50 \mathrm{kDa}, 40 \mathrm{kDa}$, and $30 \mathrm{kDa}$. The buffer used here was composed of $20 \mathrm{mM}$ Tris, $\mathrm{pH}$ 8.0, 1.2 M NaCl, and $10 \mathrm{mM}$ DTT. For HiLoad Superdex 200 pg 16/60 size exclusion chromatographic column $(1 \mathrm{~mL} / \mathrm{min})$, the gel phase distribution coefficient $\left(\mathrm{K}_{\mathrm{av}}\right)$ of Conalbumin $(75 \mathrm{kDa})$, Aldolose (158 kDa) and Ferritin (440 kDa, Horse Spleen) in Gel Filtration Calibration kit LMW (28-4038-42) were close to 0.38, 0.26 and 0.10, respectively.

Peak I obtained after $\mathrm{Ni}^{2+}$ affinity chromatography could be mainly eluted by SEC at three peaks, with retention times of $45.5 \mathrm{~min}, 67.2 \mathrm{~min}$, and $79.2 \mathrm{~min}$ (Fig 3A). $67.2 \mathrm{~min}$ species (gel phase distribution coefficient, $\left.\mathrm{K}_{\mathrm{av}}, 0.37\right)$ is approximate to Conalbumin $(75 \mathrm{kDa}, 0.36)$ and the theoretical molecular weight of Sup35 monomer is $76.6 \mathrm{kDa}$. BN-PAGE (Fig 2C) and SEC curve (Fig 3A) stated that peak I contains Sup35 monomer $\left(67.2 \mathrm{~min}, \mathrm{~K}_{\mathrm{av}}, 0.37\right)$ and a protein complex $\left(45.5 \mathrm{~min}, \mathrm{~K}_{\mathrm{av}}, 0.11\right)$, which is an intact complex and not a mixture. This determined 45.5 min does not possess large complexes which all run in the void volume at the same time. Fractionated samples were dissociated into zone X2 bands (red frame in Fig 3B), Sup35 monomer band (black frame in Fig 3B), and X1 band (blue frame in Fig 3B). X1 was identified as ATP synthase $\beta$ unit (chain D) by mass spectroscopy and zone X2 contained Sup35, chaperone HSP90, Dna K (HSP70), $30 \mathrm{~S}$ subunit (Chain C) of E. coli $70 \mathrm{~S}$ ribosome, and Omp F (Table S1). SDS-PAGE for 45.5 
min (Fig 3B) and further mass spectrum (Table S1) all pointed out that Sup35 protein exists in 45.5 min complex.

Previous studies $[27,28]$ had revealed that Sup35-NM could aggregate through self-assembly into amyloid fibrils, which are relatively high molecular weight polymers featured as smear bands in SDD-AGE. The 45.5 min species had a smear SDD-AGE band, which is fundamentally different from that exhibited by the 67.2 min monomer (Fig 3C). This band pattern elucidated that 45.5 min has aggregated Sup35 (C-aggregates) rather than Sup35 monomers bounded with other protein complexes. Take together, the 45.5 species was assigned as a complex, which was formed by combining Sup35 C-aggregates and zone X2 proteins in E. coli

The 45.5 min species showed the identical aggregation pattern with Sup35-NM fibril. However, the TEM image of the $45.5 \mathrm{~min}$ species had a distinct outlook compared with purified Sup35 fibrils (Fig 3D) [22,28]. HSP90-Dna K-client protein complex was activated during protein misfolding or aggregation, and yeast Ssa1p (HSP90) could inhibit Sup35 monomers to form fibrils [28,29-32]. Here, peak I could be divided in three parts; one was determined to be Sup35 C-aggregate-HSP90-Dna K-30 S ribosomal subunit-Omp F due to the interactions of aggregated Sup35 client with HSP90-Dna K, and the others were ATP synthase $\beta$ unit (chain D) and Sup35 monomers. The distinctive Sup35 folding states in Sup35 C-aggregate and Sup35 monomer were inherited from the states in $E$. coli rather than man-made due to the protein purification process. This suggests that HSP90-Dna K could interact with Sup35 aggregates formed in E. coli. Through the influence of Sup35 C-aggregate-HSP90-Dna K-30S ribosomal subunit-Omp F complex on Sup35 purification, we could obtain a portion of Sup35 monomer and ATP synthase using SEC. The Sup35 monomer identified here coincided with some Sup35 monomers dissociated from peak I in BN-PAGE (Fig 2C). All these proofs demonstrate that Sup35 C-aggregate possessed a different in vivo aggregate manner with in vitro -prepared amyloid fibrils.

Fractionated Sup35 monomer could not form an aggregate pattern identical to Sup35 C-aggregate and Sup35-NM fibril. Sup35 monomer could maintain its conformational stability in vitro for a long time and showed an SDD-AGE pattern similar to that observed from the yeast [psi ${ }^{-}$] monomer state Sup35 (Fig $4 \mathrm{~A}$ ), which indicates that Sup35 monomer was expressed as a conformationally stable species as opposed to Sup35 C-aggregates. They did not possess the ability to form fibrils without being accompanied by the ATP regeneration system [22,33]. The 65.5 min species $\left(\mathrm{K}_{\mathrm{av}}, 0.35\right)$ was Sup35 monomer and had the same protein state with $67.2 \mathrm{~min}$ monomers (Figure $3 \mathrm{~A}$ ). The $\mathrm{K}_{\mathrm{av}}$ of 55 min species was 0.22 , which was between 0.10 (Aldolose, $158 \mathrm{kDa}$ ) and 0.26 (Ferritin, $44 \mathrm{kDa}$ ). And, we had previous found that Sup35-NM could form trimer [28]. 55 min species could be low molecular weight aggregates of purified in vitro Sup35 monomers, such as Sup35 trimers (230 kDa). A small amount of the 14-day Sup35 monomer had 55 min species (Fig 4B), which suggested that in vitro Sup35 monomer has a tendency for aggregation, and perhaps the protection system functioned to avoid heterologous prion assembly in E. coli. Sup35 C-aggregates had the 43.5 min retention time after 14-day of storagein vitro (Fig S2), which indicates that the Sup35 C-aggregate-HSP90Dna K-30 S ribosomal subunit-Omp F complex was not in a state of equilibrium and that this complex blocks Sup35 aggregates from further propagation. This suggests that the Sup35 C-aggregate state fold may be a foreign species in E. coli, which was monitored under the surveillance of the HSP90-Dna K chaperone system. 

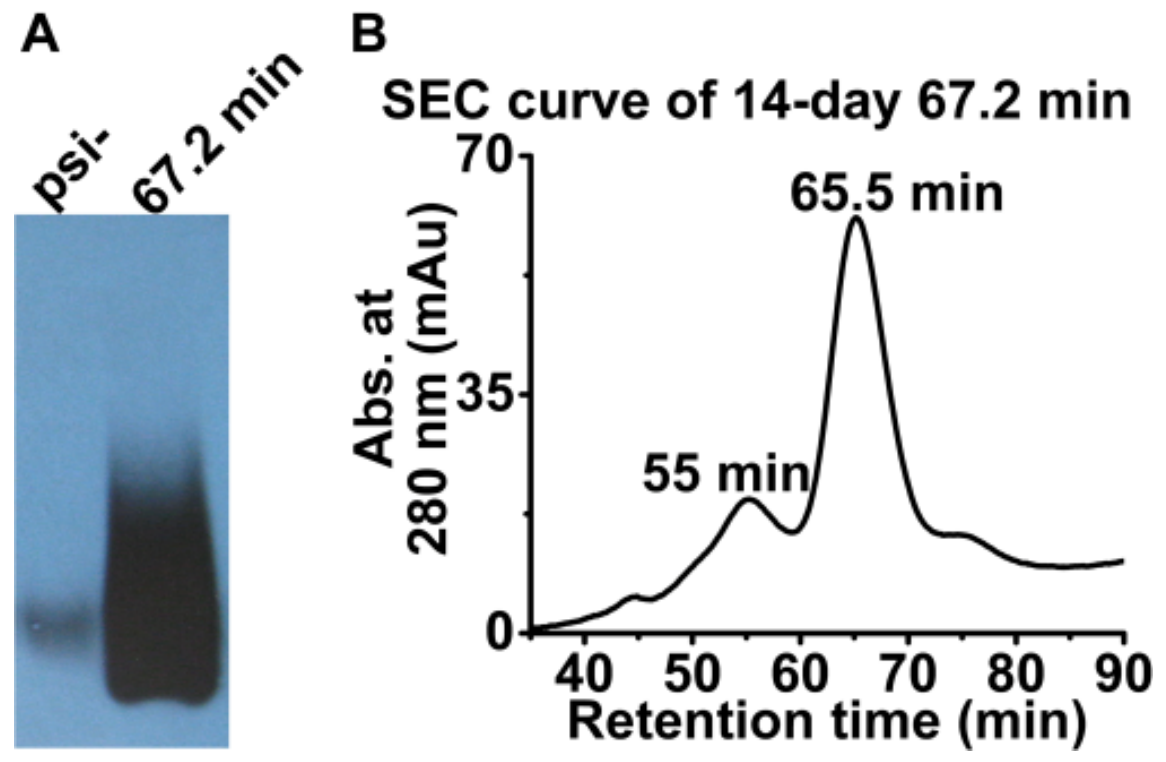

Fig 4. Long-term storage of Sup35 monomer. (A) SDD-AGE of [psi- $]$ in vivo Sup35 monomer and $E$. coli 67.2 min monomer species stored for 14 days in vitro . psi- and $67.2 \mathrm{~min}$ had the same amount of total proteins and 67.2 min species had more Sup35 proteins than psi- due to the background expression in psicells. (B) SEC curve of 14-day 67.2 min speciesin vitro . E. coli Sup35 monomer in vitro was stored for 14 days at $4{ }^{\circ} \mathrm{C}$. The buffer used here was composed of $20 \mathrm{mM}$ Tris, $\mathrm{pH} 8.0,1.2 \mathrm{M} \mathrm{NaCl}$, and $10 \mathrm{mM}$ DTT.

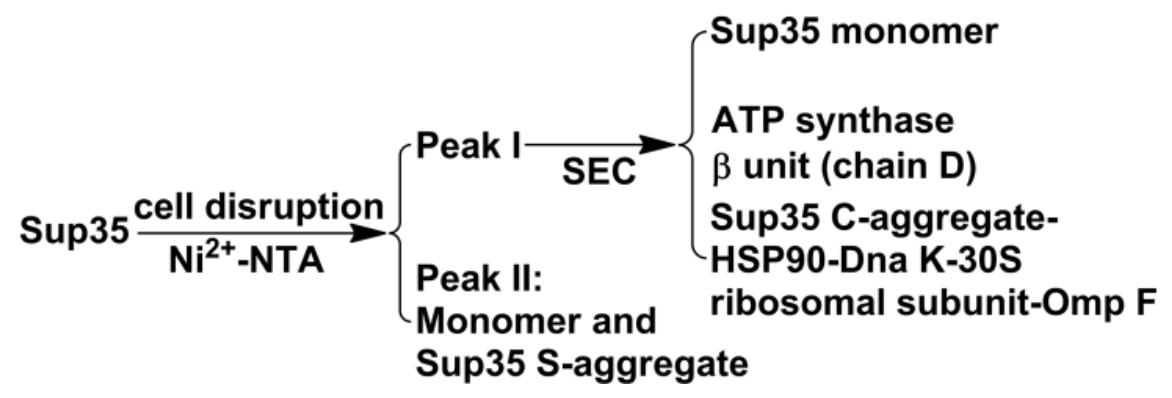

Fig 5. Identification of three Sup35 states in E. coli.

In summary, Sup35 aggregates could form a complex with cytoplasmic HSP90 and Dna K in E. coli, and a stable monomer also existed (Figure 5). The aggregate state was maintained by a complex, which was composed of non-equilibrium bindings of molecular chaperones. In addition, the Sup35 complex exhibited an aggregate morphology different from the Sup35 fibrils formed in vitro . Further studies are underway to elucidate the different regulatory mechanisms underlying prion assembly in E. coli and yeast.

Supporting Information

Fig S1. pET28a-Sup35 vector map.

Fig S2. SEC curve of 14-day 45.4 min species in vitro.45.4 min species fractioned in Figure $3 \mathrm{~A}$ were stored for 14 days at $4^{\circ} \mathrm{C}$. The buffer used here were $20 \mathrm{mM}$ Tris, $\mathrm{pH}$ 8.0, $1.2 \mathrm{M} \mathrm{NaCl}$, and $10 \mathrm{mM}$ DTT.

Table S1. Mascot search mass spectrum results of bands in the red frame (zone X2), blue frame (X1), and black frame (Sup35 monomer band) in Fig 3B.

Competing Interests 
The authors have declared that no competing interests exist.

\section{Author Contributions}

All authors confirmed they have contributed to the intellectual content of this paper and have met the following 4 requirements: (a) significant contributions to the conception and design, acquisition of data, or analysis and interpretation of data; (b) drafting or revising the article for intellectual content; (c) final approval of the published article; and (d) agreement to be accountable for all aspects of the article thus ensuring that questions related to the accuracy or integrity of any part of the article are appropriately investigated and resolved.

\section{Research funding}

This work was supported by 2019 Medical Science and Technology Research Project of Henan Province (No. SB201902009). The funding organization(s) played no role in the study design; in the collection, analysis, and interpretation of data; in the writing of the report; or in the decision to submit the report for publication.

\section{References}

1. Morimoto, RI. Proteotoxic stress and inducible chaperone networks in neurodegenerative disease and aging. Genes Dev 2008;22:1427-1438.

2. Kim YE, Hipp MS, Bracher A, Hayer-Hartl M, Hartl, FU. Molecular chaperone functions in protein folding and proteostasis. Annu Rev Biochem 2013;82: 323-355.

3. Li Y, Yan J, Zhang X, Huang K. Disulfide bonds in amyloidogenesis diseases related proteins. Proteins 2013;81:1862-1873.

4. Avni A, Swasthi HM, Majumdar A, Mukhopadhyay S. Intrinsically disordered proteins in the formation of functional amyloids from bacteria to humans. Progress in Molecular Biology and Translational Science 2019;166:109-143.

5. Wickner RB, Shewmaker FP, Bateman DA, Edskes HK, Gorkovskiy A, Dayani Y, Bezsonov EE. Yeast prions: structure, biology, and prion-handling systems. Microbiol Mol Biol Rev 2015;79:1-17.

6. Guo JL, Lee VM. Cell-to-cell transmission of pathogenic proteins in neurodegenerative diseases. Nat Med 2014;20:130-138.

7. Sandberg MK, Al-Doujaily H, Sharps B, Clarke AR, Collinge J. Prion propagation and toxicity in vivo occur in two distinct mechanistic phases. Nature 2011;470:540-542.

8. Kiktev DA, Melomed MM, Lu CD, Newnam GP, Chernoff YO. Feedback control of prion formation and propagation by the ribosome-associated chaperone complex. Mol Microbiol 2015:96:621-632.

9. Tanaka M, Chien P, Naber N, Cooke R, Weissman JS. Conformational variations in an infectious protein determine prion strain differences. Nature 2004:428:323-328.

10. Liu Y, Wei H, Wang J, Qu J, Zhao W, Tao H. Effects of randomizing the Sup35NM prion domain sequence on formation of amyloid fibrils in vitro, Biochem Biophys Res Commun 2007:353:139-146.

11. Tanaka M, Weissman JS. An efficient protein transformation protocol for introducing prions into yeast. Methods Enzymol 2006:412:185-200.

12. Fink, GR. A transforming principle. Cell 2005;120:153-154.

13. Kryndushkin DS, Alexandrov IM, Ter-Avanesyan MD, Kushnirov VV. Yeast[PSI+] prion aggregates are formed by small Sup35 polymers fragmented by Hsp104, J Biol Chem 2003;278:49636-49643.

14. Shorter J, Lindquist S. Hsp104 catalyzes formation and elimination of self-replicating Sup35 prion conformers. Science 2004;304:1793-1797. 15. Ohta S, Kawai-Noma S, Kitamura A, Pack CG, Kinjo M, Taguchi H. The interaction of Hsp104 with yeast prion Sup35 as analyzed by fluorescence cross-correlation 
spectroscopy. Biochem Biophys Res Commun 2013;442:28-32. 16. Tessier PM, Lindquist S. Unraveling infectious structures, strain variants and species barriers for the yeast prion [PSI+]. Nat Struct Mol Biol 2009;16:598-605. 17. Ohhashi Y, Ito K, Toyama BH, Weissman JS, Tanaka M. Differences in prion strain conformations result from non-native interactions in a nucleus, Nat Chem Biol 2010;6:225-230. 18. Krishnan R, Goodman JL, Mukhopadhyay S, Pacheco CD, Lemke EA, Deniz A A, Lindquist S. Conserved features of intermediates in amyloid assembly determine their benign or toxic states. Proc Natl Acad Sci 2012;109:1117211177. 19. Park S, Wang X, Xi W, Richardson R, Laue TM, Denis CL. The non-prion SUP35 preexists in large chaperone-containing molecular complexes. Proteins 2021;1- 12. 20. Marshall KE, Offerdahl DK, Speare JO, Dorward DW, Hasenkrug AB, Carmody AB, Baron GS. Glycosylphosphatidylinositol anchoring directs the assembly of Sup35NM protein into non-fibrillar, membrane-bound aggregates. J Bio Chem 2014: 289:12245-12263. 21. Garrity SJ, Sivanathan V, Dong J, Lindquist S, Hochschild A. Conversion of a yeast prion protein to an infectious form in bacteria. Proc Natl Acad Sci 2010;107: 10596-10601. 22. Krzewska J, Melki R. Molecular chaperones and the assembly of the prion Sup35p, an in vitro study, EMBO J 2006;25:822-833. 23. Krzewska J, Tanak, M, Burston SG, Melki R. Biochemical and functional analysis of the assembly of full-length Sup35p and its prion-forming domain. J Biol Chem 2007;282:16791686. 24. Horstman AL, Kuehn MJ. Enterotoxigenic Escherichia colisecretes active heat-labile enterotoxin via outer membrane vesicles. J Biol Chem 2000;275: 12489-12496. 25. Chien P, DePace AH, Collins SR, Weissman JS. Generation of prion transmission barriers by mutational control of amyloid conformations. Nature 2003;424;948-951. 26. Wang MY, Zhang FF, Song C, Shi PF, Zhu J. A Decentralized Approach to the Formulation of Hypotheses: A Hierarchical Structural Model for a Prion Self-Assembled System. Sci Rep 2016;6. 27. Lin J, Lucius AL. Examination of ClpB Quaternary Structure and Linkage to Nucleotide Binding. Biochemistry 2016;55:1758-1771. 28. Genest O, Hoskins JR, Kravats AN, Doyle SM, Wickner S. Hsp70 and Hsp90 of E. coli Directly Interact for Collaboration in Protein Remodeling. J Mo. Biol 2015; 427:3877-3889. 29. Freitag DG, Ouimet PM, Girvitz TL, Kapoor M. Heat shock protein 80 of Neurospora crassa, a cytosolic molecular chaperone of the eukaryotic stress 90 family, interacts directly with heat shock protein 70, Biochemistry 1997;36:10221-10229. 30. Taipale M, Jarosz DF, Lindquist S. HSP90 at the hub of protein homeostasis: emerging mechanistic insights. Nat Rev Mol Cell Biol 2010;11:515-528. 31. Shorter J, Lindquist S. Destruction or potentiation of different prions catalyzed by similar Hsp104 remodeling activities. Mol Cell 2006;23:425-438.

Figure Legends

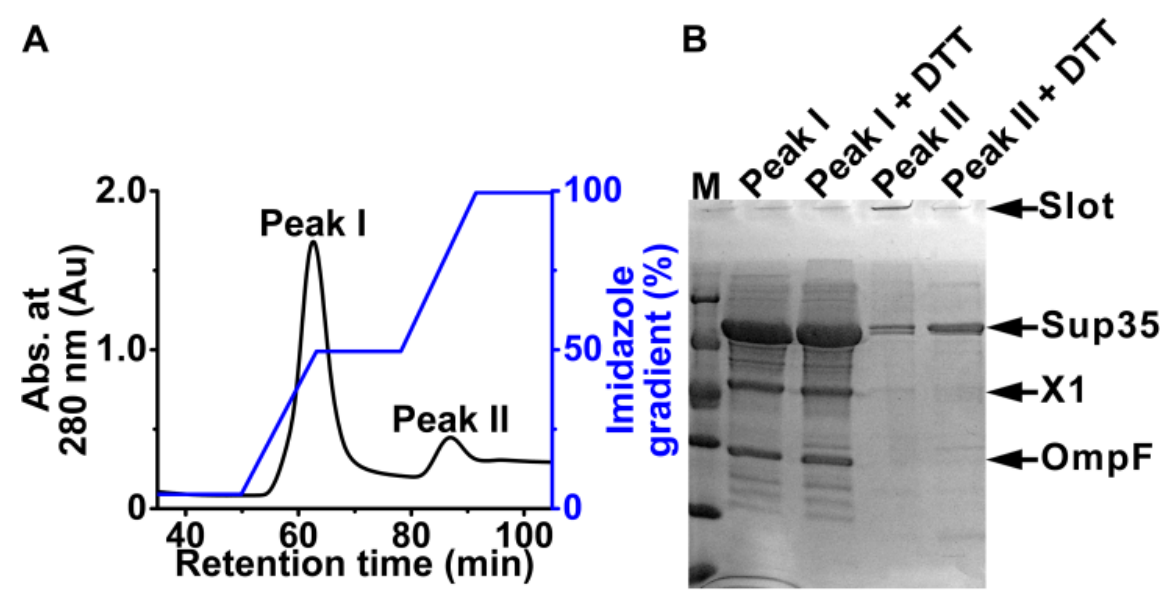

Fig 1. Purification and SDS-PAGE of overexpressed Sup35 in E. coli under non-denatured $\mathrm{Ni}^{2+}$ affinity chromatography. (A) Elution curve of Sup35 under non-denatured $\mathrm{Ni}^{2+}$ affinity chromatography. Peak I and peak II appeared in approximately 5-50\% and 50-100\% imidazole gradients, respectively. The buffers used here were buffer A (20 mM Tris, pH 8.0, 1.2 M NaCl, $10 \mathrm{mM}$ PMSF, 0\% imidazole) and buffer B (20 mM Tris, pH 8.0, 1.2 M NaCl, $10 \mathrm{mM} \mathrm{PMSF}, 500 \mathrm{mM}$ imidazole, 100\%). (B) SDS-PAGE for the fractions 
in Figure 1A. All lanes were treated by SDS boiling. Slot represents for sample loading slots in stacking gel. Sup35, X1 and OmpF indicate the bands of Sup35 monomer, X1 protein and OmpF, respectively. Peak $\mathrm{I}+$ DTT and Peak II + DTT were boiled with $10 \mathrm{mM}$ DTT. Lane M: molecular weight protein marker comprising $116 \mathrm{kDa}, 66.2 \mathrm{kDa}, 45 \mathrm{kDa}, 35 \mathrm{kDa}, 25 \mathrm{kDa}$, and $18.4 \mathrm{kDa}$.

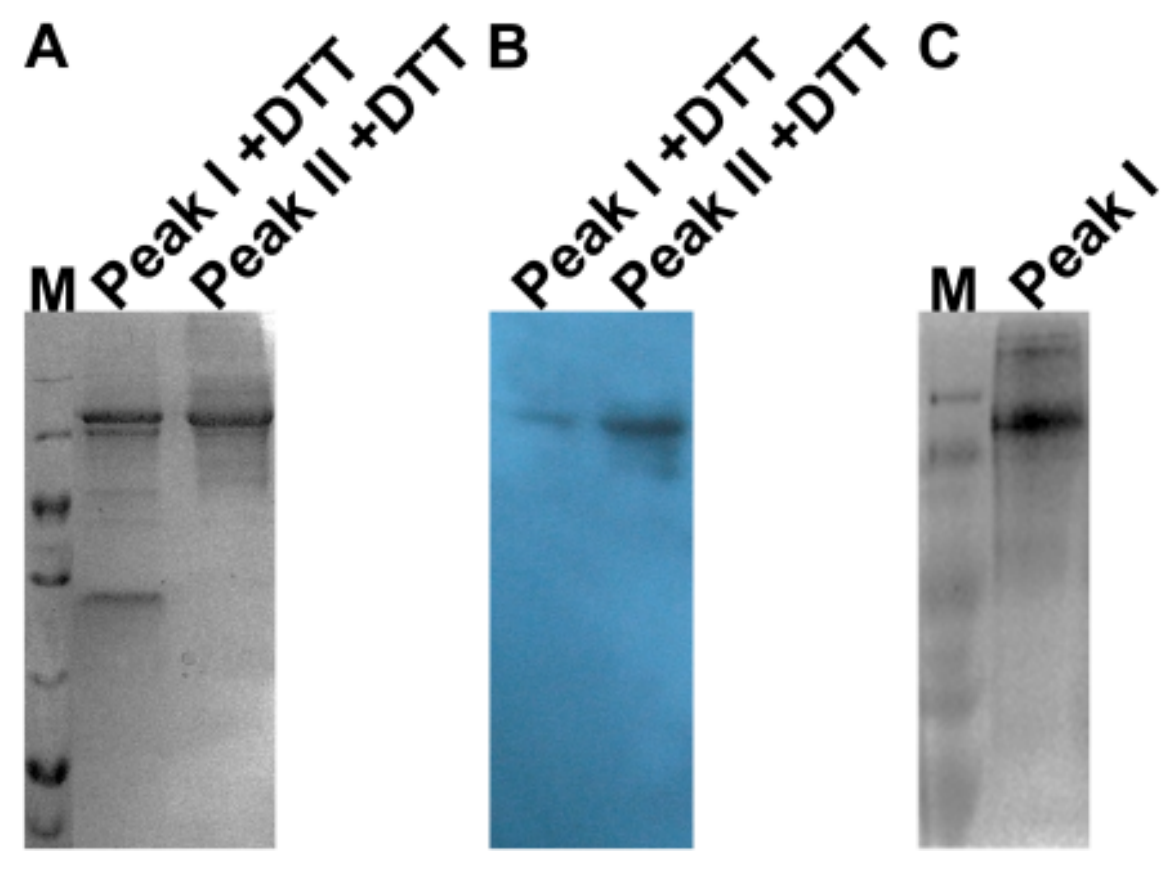

Fig 2. Western blot and BN-PAGE analyses of peak I and II. (A) SDS-PAGE of peak I (lane 1) and peak II (lane 2) under DTT. (B) Western blot of peak I and peak II, with DTT, using Sup35 sequencespecific antibody. (C) BN-PAGE analysis of peak I. Peak I + DTT and Peak II + DTT all had the same amount of total protein in Figures $2 \mathrm{~A}$ and $2 \mathrm{~B}$. The amount of protein in Figure $2 \mathrm{C}$ was twice as much as that in Figure 2A. Lanes $\mathrm{M}$ in Figures $2 \mathrm{~A}$ and $2 \mathrm{C}$ : molecular weight protein marker comprising $116 \mathrm{kDa}$, $66.2 \mathrm{kDa}, 45 \mathrm{kDa}, 35 \mathrm{kDa}, 25 \mathrm{kDa}, 18.4 \mathrm{kDa}$, and $14.4 \mathrm{kDa}$. 

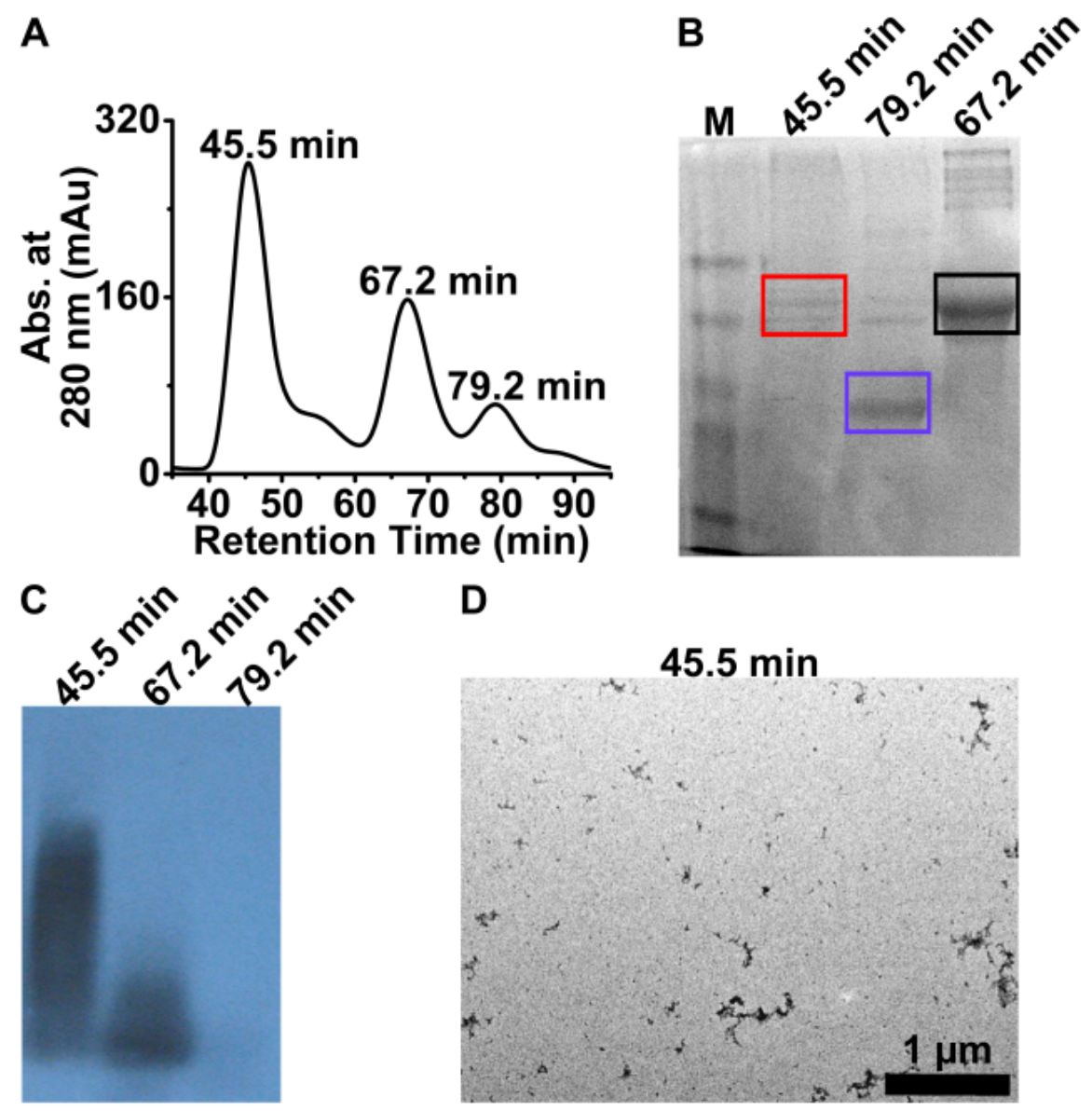

Fig 3. Dissection of peak I and subsequent analysis.(A) Size exclusion chromatography (SEC) curve of peak I. Imidazole was removed from the peak I sample using dialysis. $45.5 \mathrm{~min}, 67.2 \mathrm{~min}$, and $79.2 \mathrm{~min}$ represented the different retention times of the fractionated species. Buffer $\mathrm{C}$ was used here $(20 \mathrm{mM}$ Tris, $\mathrm{pH}$ 8.0, 1.2 M NaCl). (B) SDS-PAGE for $45.5 \mathrm{~min}, 67.2 \mathrm{~min}$, and $79.2 \mathrm{~min}$ fractions in Figure 3A. The bands in black, blue, and red frames were selected for mass spectrometry. (C) SDD-AGE of peak I SEC fractions. (D) Transmission electron microscopy (TEM) of 45.5 min species from Figure 3A. Lane $\mathrm{M}$ in Figure 3B: molecular weight protein marker comprising $100 \mathrm{kDa}, 70 \mathrm{kDa}, 50 \mathrm{kDa}, 40 \mathrm{kDa}$, and $30 \mathrm{kDa}$. The buffer used here was composed of $20 \mathrm{mM}$ Tris, $\mathrm{pH} 8.0,1.2 \mathrm{M} \mathrm{NaCl}$, and $10 \mathrm{mM}$ DTT. For HiLoad Superdex 200 pg 16/60 size exclusion chromatographic column $(1 \mathrm{~mL} / \mathrm{min})$, the gel phase distribution coefficient $\left(\mathrm{K}_{\mathrm{av}}\right)$ of Conalbumin $(75 \mathrm{kDa})$, Aldolose (158 kDa) and Ferritin (440 kDa, Horse Spleen) in Gel Filtration Calibration kit LMW (28-4038-42) were close to 0.38, 0.26 and 0.10 , respectively. 

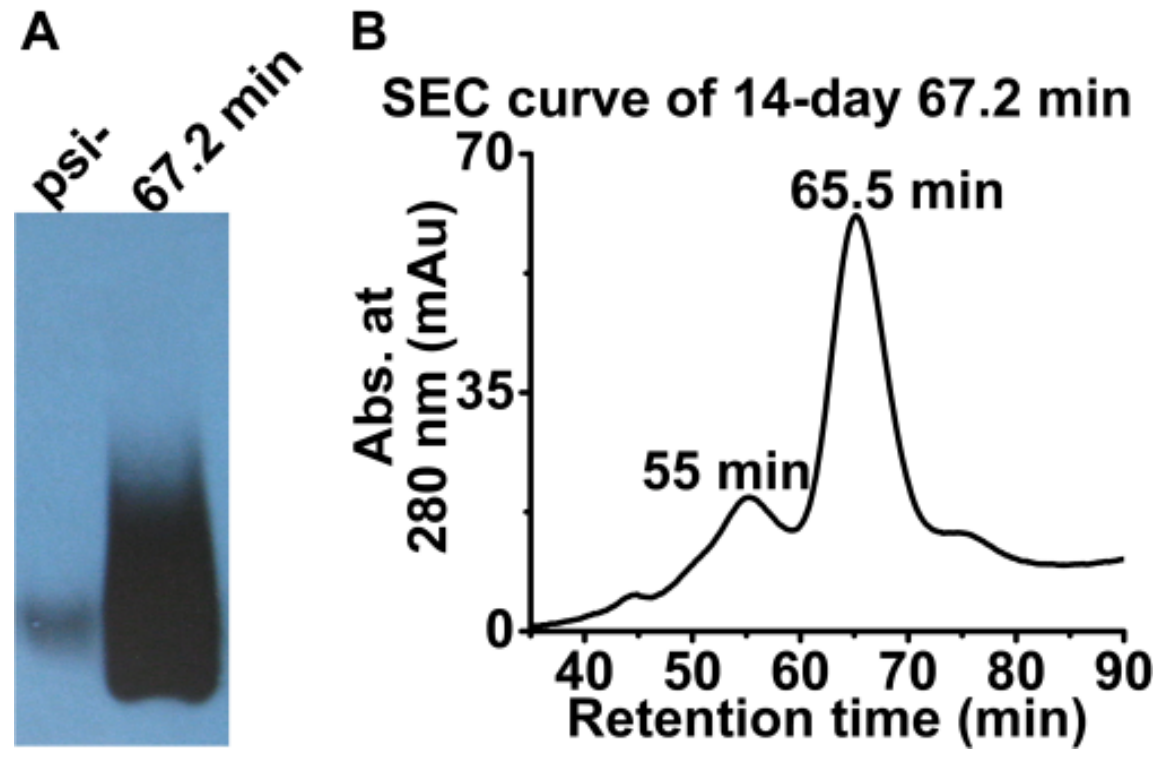

Fig 4. Long-term storage of Sup35 monomer. (A) SDD-AGE of [psi $i^{-}$in vivo Sup35 monomer andE. coli 67.2 min monomer species stored for 14 days in vitro . psi- and $67.2 \mathrm{~min}$ had the same amount of total proteins and 67.2 min species had more Sup35 proteins than psi- due to the background expression in psicells. (B) SEC curve of 14-day 67.2 min speciesin vitro . E. coli Sup35 monomer in vitro was stored for 14 days at $4{ }^{\circ} \mathrm{C}$. The buffer used here was composed of $20 \mathrm{mM}$ Tris, pH 8.0, 1.2 M NaCl, and $10 \mathrm{mM}$ DTT.

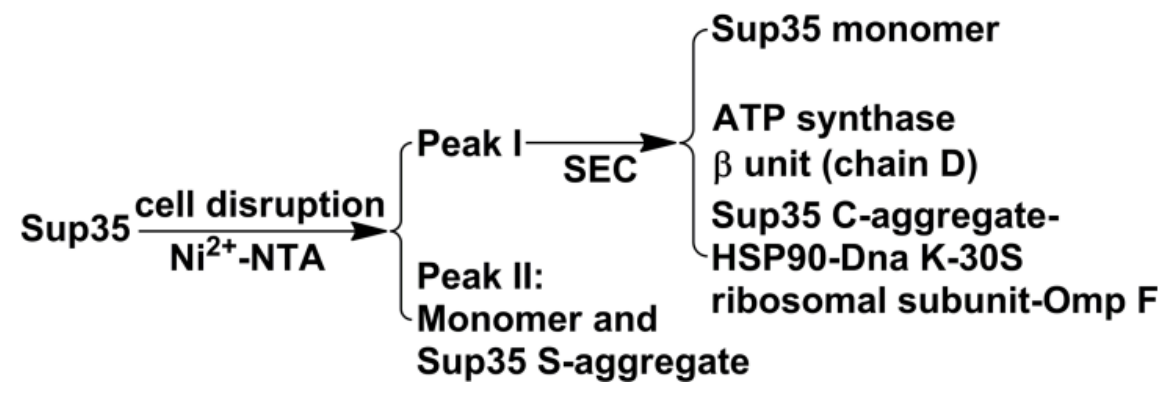

Fig 5. Identification of three Sup35 states in E. coli. 


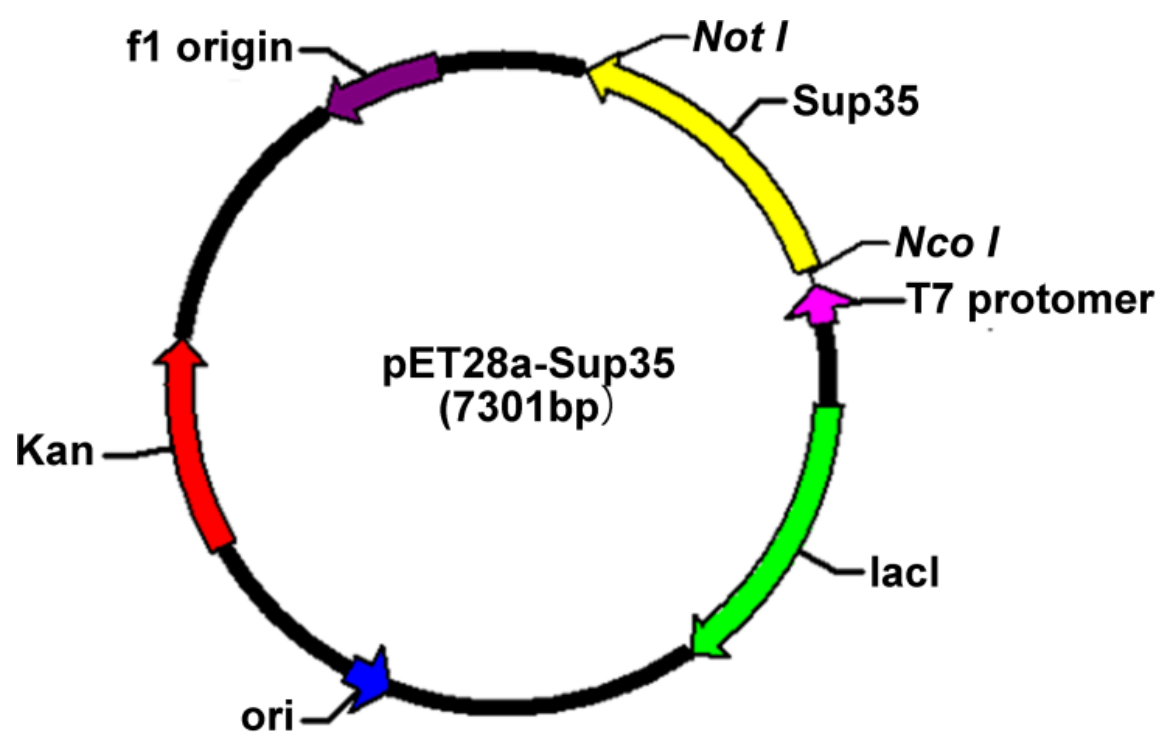

Fig S1. pET28a-Sup35 vector map.

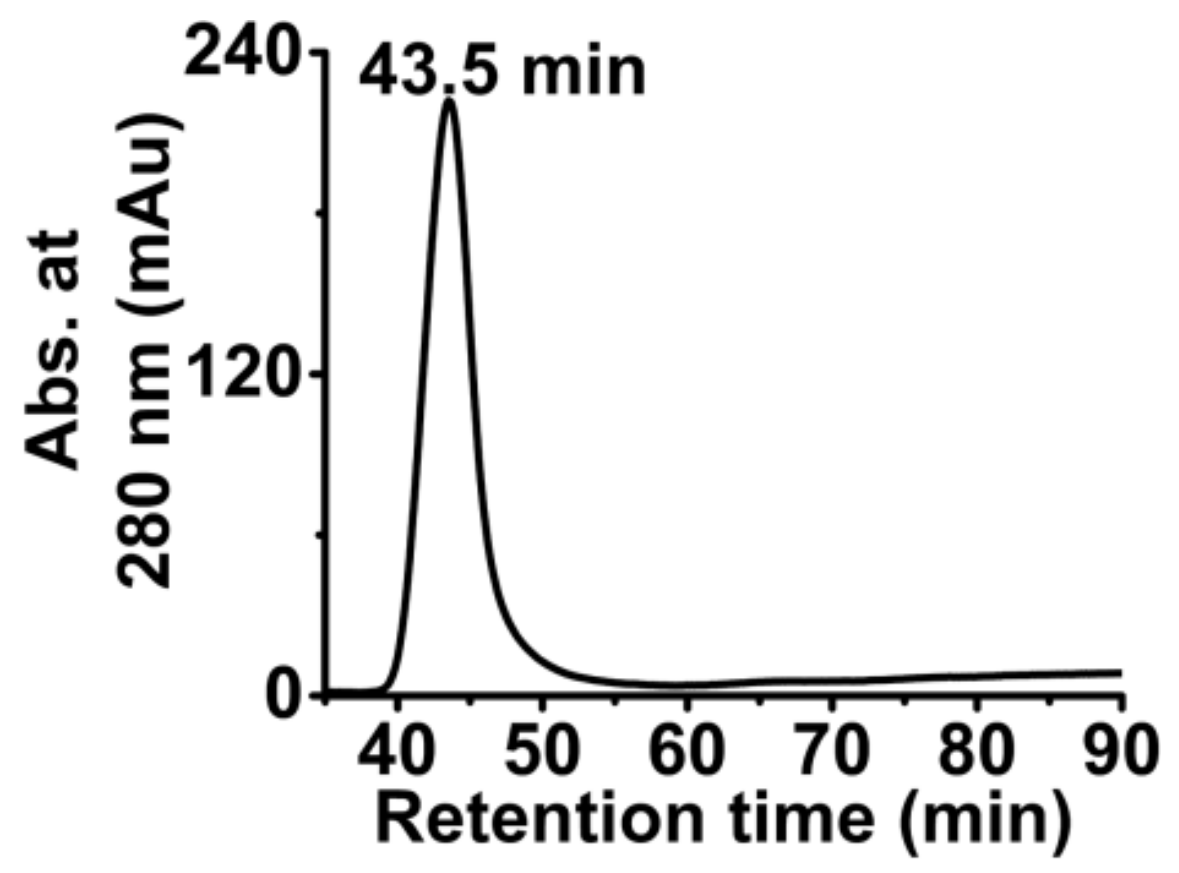

Fig S2. SEC curve of 14-day $\mathbf{4 5 . 4}$ min species in vitro.45.4 min species fractioned in Figure 3A were stored for 14 days at $4^{\circ} \mathrm{C}$. The buffer used here were $20 \mathrm{mM}$ Tris, $\mathrm{pH} 8.0,1.2 \mathrm{M} \mathrm{NaCl}$, and $10 \mathrm{mM}$ DTT.

Table S1. Mascot search mass spectrum results of bands in the red frame (zone X2), blue frame (X1), and black frame (Sup35 monomer band) in Fig 3B.

Acknowledgements 
Table S1. Mascot search mass spectrum results of bands in the red frame (zone X2), blue frame (X1), and black frame (Sup35 monomer band) in Figure 3B.

\begin{tabular}{|c|c|c|c|}
\hline & zone $\mathrm{X} 2$ & Sup35 & $\mathrm{X} 1$ \\
\hline Database & $\begin{array}{lr}\text { NCBInr } & 20141208 \\
(53438708 & \begin{array}{r}\text { sequences; } \\
19231914498 \\
\text { residues })\end{array}\end{array}$ & $\begin{array}{l}\text { NCBInr 20141208 } \\
\text { (53438708 } \\
\text { sequences; } \\
\text { 19231914498 } \\
\text { residues) }\end{array}$ & $\begin{array}{l}\text { NCBInr 20141208 } \\
\text { (53438708 } \\
\text { sequences; } \\
\text { 19231914498 } \\
\text { residues) }\end{array}$ \\
\hline Taxonomy & E. coli (1254256 sequences) & $\begin{array}{l}\text { S. cerevisiae } \\
(50241 \\
\text { sequences })\end{array}$ & $\begin{array}{l}\text { E. coli }(1254256 \\
\text { sequences) }\end{array}$ \\
\hline Protein hits & 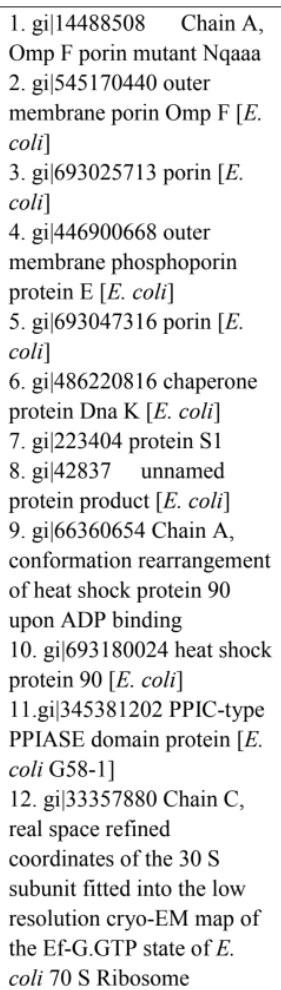 & $\begin{array}{l}\text { 1. gi } \mid 618713641 \\
\text { Sup35[S. } \\
\text { cerevisiae }] \\
\text { 2. gi } \mid 3712 \text { GST1 } \\
{[\text { S. cerevisiae }]} \\
\text { 3. gi } \mid 618713585 \\
\text { Sup35 }[S \text {. } \\
\text { cerevisiae }]\end{array}$ & $\begin{array}{l}\text { 1. gi|333944305 } \\
\text { Chain D, structure } \\
\text { of The } E \text {. coli } \\
\text { F1-ATP synthase } \\
\text { inhibited by } \\
\text { subunit epsilon } \\
\text { gi|672830493 } \\
\text { 2. ATP synthase } \\
\text { F0F1 subunit beta, } \\
\text { partial }[\text { E. coli }]\end{array}$ \\
\hline
\end{tabular}

